ACCEPTED BY APJ

Preprint typeset using $\mathrm{IATEX}_{\mathrm{E}} \mathrm{X}$ style emulateapj v. 08/22/09

\title{
THE CLUSTERING BEHAVIOR OF PRE-MAIN SEQUENCE STARS IN NGC 346 IN THE SMALL MAGELLANIC CLOUD
}

\author{
Stefan SchmeJa \\ Zentrum für Astronomie der Universität Heidelberg, Institut für Theoretische Astrophysik, Albert-Ueberle-Str. 2, 69120 Heidelberg, \\ Germany \\ Dimitrios A. Gouliermis \\ Max-Planck-Institut für Astronomie, Königstuhl 17, 69117 Heidelberg, Germany \\ AND \\ RALF S. KLESSEN \\ Zentrum für Astronomie der Universität Heidelberg, Institut für Theoretische Astrophysik, Albert-Ueberle-Str. 2, 69120 Heidelberg, \\ Germany \\ accepted by ApJ
}

\begin{abstract}
We present evidence that the star-forming region NGC 346/N66 in the Small Magellanic Cloud is the product of hierarchical star formation, probably from more than one star formation event. We investigate the spatial distribution and clustering behavior of the pre-main sequence (PMS) stellar population in the region, using data obtained with Hubble Space Telescope's Advanced Camera for Surveys. By applying the nearest neighbor and minimum spanning tree methods on the rich sample of PMS stars previously discovered in the region we identify ten individual PMS clusters in the area and quantify their structures. The clusters show a wide range of morphologies from hierarchical multipeak configurations to centrally condensed clusters. However, only about 40 per cent of the PMS stars belong to the identified clusters. The central association NGC 346 is identified as the largest stellar concentration, which cannot be resolved into subclusters. Several PMS clusters are aligned along filaments of higher stellar density pointing away from the central part of the region. The PMS density peaks in the association coincide with the peaks of [O III] and $8 \mu \mathrm{m}$ emission. While more massive stars seem to be concentrated in the central association when considering the entire area, we find no evidence for mass segregation within the system itself.
\end{abstract}

Subject headings: stars: formation — stars: pre-main sequence — galaxies: star clusters — Magellanic Clouds - Methods: statistical

\section{INTRODUCTION}

Star formation is generally thought to be clumped into a hierarchy of structures, from small multiple systems to giant stellar complexes and beyond (e.g. Efremov \& Elmegreen 1998), and the interaction between gravity and supersonic turbulent motions has been proposed as a major player in the formation of hierarchical structures (Mac Low \& Klessen 2004; Elmegreen 2006; Ballesteros-Paredes et al. 2007; McKee \& Ostriker 2007). The interstellar medium has also been observed to be arranged in a hierarchical structure (Scalo 1985; Vázquez-Semadeni 2004), sometimes described as fractal, from the scales of the largest giant molecular clouds (GMCs) down to individual cores and embedded clusters, which are sometimes hierarchical themselves.

The velocity structure in such turbulent regions is suggested to be scale-free, meaning that the same physical processes and the same velocity-separation relations occur over a wide range of absolute scales (e.g. Elmegreen 2006). According to this hypothesis, large clumps originate from the large velocities that compress the gas on large scales, and small clumps result from the compression of the gas characterized by small velocities on small

Electronic address: sschmeja@ita.uni-heidelberg.de

Electronic address: dgoulier@mpia.de

Electronic address: rklessen@ita.unı-heidelberg.de scales. Considering that stars are formed by the collapse of such hierarchical structured clumps, they are themselves hierarchically structured. Indeed, stars are mostly formed in groups (e.g. Stahler \& Palla 2005), which can be seen as the bottom parts of this hierarchy (e.g. Elmegreen et al. 2000).

Naturally, the structure of such systems, i.e. the spatial distribution of their members, may hold important information on the mechanism and the initial conditions of their formation. However, a thorough investigation of the structural behavior of star formation in environments different than our Galaxy was not possible, until recently, due to observational limitations. In this paper we present such an investigation for the brightest starforming region in the Small Magellanic Cloud (SMC).

LHA 115-N66 or in short N66 (Henize 1956) is a very active star-forming region in the SMC with a diameter of more than $9^{\prime}$ at a distance of about $60 \mathrm{kpc}$ (e.g. Laney \& Stobie 1994). This H II region is also known as DEM S 103 (Davies et al. 1976) or NGC 346 referring to the bright stellar association located in its center. Being the largest and most luminous H II region in the SMC, NGC 346/N66 has been mapped in several wavelengths. Observations of its area have been taken in the Xrays with XMM-Newton and Chandra (Nazé et al. 2002, 2004), in the UV with the International Ultraviolet Ex- 


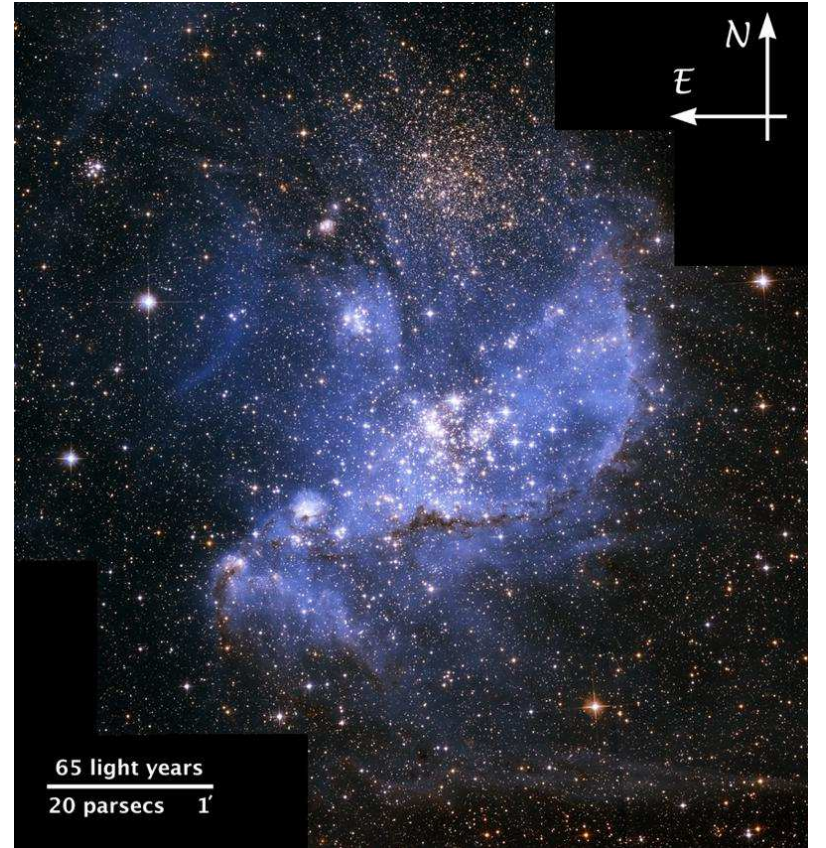

FIG. 1.- Color-composite image of the three partially overlapping ACS/WFC observed fields of NGC 346, covering a total area of $5^{\prime} \times 5^{\prime}$. North is up, and east is to the left. Image Credits: NASA, ESA and A. Nota (STScI/ESA).

plorer (de Boer \& Savage 1980) and the Far-Ultraviolet Spectroscopic Explorer (Danforth et al. 2003), in the $\mathrm{CO}(2-1)$ line with the SEST telescope (Contursi et al. 2000), and in the infrared with the Infrared Space Observatory (Contursi et al. 2000) and more recently with the Spitzer Space Telescope (Bolatto et al. 2007; Simon et al. 2007). The region has also been covered by observations of the radio continuum (Ye et al. 1991; Reid et al. 2006) and of the 21-cm atomic line (Staveley-Smith et al. 1997). Maps of the ionized gas have also been made in $\mathrm{H} \alpha$ (e.g. Kennicutt 1988; Le Coarer et al. 1993), and [O III] with NTT (e.g. Rubio et al. 2000).

N66 is characterized by weak molecular emission and is also deficient in H I. It hosts, though, a variety of discrete $\mathrm{H}_{2}$ emission peaks, which coincide with the main features of the ionized gas, and with compact embedded young clusters, where candidate young stellar objects (YSOs) have been identified. The central part of the whole $\mathrm{H}$ II region is characterized by an oblique bright emission region, extending from southeast to northwest, known as the bar of N66 (Fig. 1). This area is dominated by a strong UV radiation field related to the central association NGC 346. Indeed this system hosts the largest sample of spectroscopically confirmed OB stars in the whole SMC (Massey et al. 1989), which have been the subject of several previous investigations (e.g. Niemela et al. 1986; Walborn et al. 2000; Evans et al. 2006; Hunter et al. 2008). NGC 346 ionizes the remaining molecular cloud to the southwest of the bar, triggering the development of a photo-dissociated region seen as a curved front eating into the cloud.

Recent observations with the Advanced Camera for Surveys (ACS) onboard the Hubble Space Telescope (HST) provided substantial information on the stellar content of this magnificent star-forming region by revealing a plethora of low-mass pre-main sequence
(PMS) stars. This discovery clearly indicates that NGC 346/N66 also hosts low-mass star formation. Our photometric data set delivers more than 98000 stars in total, with almost 8000 being low-mass PMS stars (Gouliermis et al. 2006). The subsequent investigation of their spatial distribution showed that these stars are not homogeneously distributed across the region, being grouped in discrete concentrations, apart from the association NGC 346. Most of these "subclusters" are found to coincide with bright $\mathrm{H} \alpha$ emission, and the $\mathrm{H}_{2}$ emission peaks (Hennekemper et al. 2008). Lowmass stars with masses $\lesssim 2-3 \mathrm{M}_{\odot}$ remain in their PMS phase for 20-30 Myr (e.g. Stahler \& Palla 2005; Briceño et al. 2007). As a consequence the rich sample of low- and intermediate-mass PMS stars, found with ACS in NGC 346/N66, offers a unique opportunity to investigate clustered star formation within a time-scale of the order of $\sim 10$ Myr and length-scales of 1 to $100 \mathrm{pc}$ in a detail never achieved before for a star-forming region outside our Galaxy.

In this paper we analyze the spatial distribution of the young PMS population unveiled by HST/ACS in the bright SMC star-forming region NGC 346/N66 and compare the results to current theoretical scenarios of star formation. For this purpose, we make use of our deep ACS photometry of this region, and apply two complementary statistical methods to identify all the compact PMS clusters and analyze their structures. The data set used here is described in Section 22 the statistical methods are explained in Section 3 and the results of our structural analysis of the PMS stars is presented in Section 4. A discussion on the star formation process revealed from our analysis is given in Section 5.

\section{DESCRIPTION OF THE DATA}

The observations used in this study, taken with the Wide-Field Channel (WFC) of ACS, and their photometry are thoroughly described by Gouliermis et al. (2006). The data were collected within the HST GO Program 10248 and were retrieved from the HST Data Archive $^{1}$. Three overlapping images were taken with ACS/WFC centered on the association NGC 346 in the filters $F 555 W(\equiv V), F 814 W(\equiv I)$ and $F 658 N(\equiv \mathrm{H} \alpha)$, covering an area of about $5^{\prime} \times 5^{\prime}(\sim 80 \mathrm{pc} \times 80 \mathrm{pc}$ at the distance of the SMC). A color-composite image of this area is shown in Fig. 1. It covers the intermediate-age cluster BS 90 (Bica \& Schmitt 1995; Rochau et al. 2007), the association NGC 346 itself, and all known components of the bright nebula N66 (Henize 1956; Davies et al. 1976). Photometry was performed using the ACS module of the package DOLPHOT ${ }^{2}$, and after eliminating bad detections based on the quality parameters of the detected sources returned from DOLPHOT, more than 98000 stars down to $V \simeq 27.5 \mathrm{mag}$ were included in the photometric catalog. This catalog is complete ( $\gtrsim 50 \%$ ) down to $V \approx 27 \mathrm{mag}$. In the present study, which deals with both low- and high-mass young populations in the area, we make use of the upper main sequence (UMS)

1 The HST Data Archive is accessible from ESO at http://archive.eso.org/cms/hubble-space-telescope-data and MAS'T' at http://archive.stsci.edu/hst/

2 The ACS mode of DOLPHOT is an adaptation of the photometry package HSTphot (Dolphin 2000). The latest version can be downloaded from http://purcell.as.arizona.edu/dolphot/ 
stars with $12 \mathrm{mag} \lesssim V \lesssim 22 \mathrm{mag}$, and the red PMS stars with $V \gtrsim 22 \mathrm{mag}$, as they have been selected by Gouliermis et al. (2006).

\section{STATISTICAL METHODS}

\subsection{Nearest Neighbor Density}

Star clusters are usually identified as regions of a certain overdensity with respect to the background stellar density. The nearest neighbor (NN) method, introduced by Casertano \& Hut (1985) based on earlier work by von Hoerner (1963), estimates the local source density $\rho_{j}$ by measuring the distance from each object to its $j$ th nearest neighbor:

$$
\rho_{j}=\frac{j-1}{S\left(r_{j}\right)} m
$$

where $r_{j}$ is the distance of a star to its $j$ th nearest neighbor, $S\left(r_{j}\right)$ the surface area with the radius $r_{j}$ and $m$ the average mass of the sources $(m=1$ when considering number densities). The value of $j$ is chosen depending on the sample size. The chosen $j$ value is correlated with the sensitivity to the density fluctuations being mapped. A small $j$ value increases the locality of the density measurements at the same time as increasing sensitivity to random density fluctuations, whereas a large $j$ value will reduce that sensitivity at the cost of losing some locality. Through the use of Monte Carlo simulations B. Ferreira \& E. A. Lada (2008, in preparation) find that a value of $j=20$ is adequate to detect clusters with about 10 to 1500 members.

The positions of the cluster centers are defined as the density-weighted enhancement centers (Casertano \& Hut 1985)

$$
x_{d, j}=\frac{\sum_{i} x_{i} \rho_{j}^{i}}{\sum_{i} \rho_{j}^{i}}
$$

where $x_{i}$ is the position vector of the $i$ th cluster member and $\rho_{j}^{i}$ the $j$ th NN density around this object.

Similarly, the density radius $r_{d}$ is defined as the density-weighted average of the distance of each star from the density center:

$$
r_{d, j}=\frac{\sum_{i}\left|x_{i}-x_{d, i}\right| \rho_{j}^{i}}{\sum_{i} \rho_{j}^{i}} .
$$

It corresponds to the observational core radius (Casertano \& Hut 1985).

We identify clusters as regions that have 20th NN density values $3 \sigma$ above the average background level.

\subsection{Minimum Spanning Tree and $\mathcal{Q}$}

The second method makes use of a minimum spanning tree (MST), a construct from graph theory, which is defined as the unique set of straight lines ("edges") connecting a given set of points without closed loops, such that the sum of the edge lengths is a minimum (Boruvka 1926; Kruskal 1956; Prim 1957). From the MST we derive the mean edge length $\ell_{\mathrm{MST}}$. Cartwright \& Whitworth (2004) introduced the parameter $\mathcal{Q}=\bar{\ell}_{\mathrm{MST}} / \bar{s}$, which combines the normalized correlation length $\bar{s}$, i.e. the mean distance between all stars, and the normalized mean edge length $\bar{\ell}_{\mathrm{MST}}$. The $\mathcal{Q}$ parameter permits to quantify the structure of a cluster and to distinguish between clusters with a central density concentration and hierarchical clusters with possible fractal substructure. Large $\mathcal{Q}$ values $(\mathcal{Q}>0.8)$ describe centrally condensed clusters having a volume density $n(r) \propto r^{-\alpha}$, while small $\mathcal{Q}$ values $(\mathcal{Q}<0.8)$ indicate clusters with fractal substructure. $\mathcal{Q}$ is correlated with the radial density exponent $\alpha$ for $\mathcal{Q}>0.8$ and anticorrelated with the fractal dimension $D$ for $\mathcal{Q}<0.8$. The dimensionless measure $\mathcal{Q}$ is independent of the number of objects and of the cluster area. A detailed description of the method, in particular its implementation and normalization used for this study, is given by Schmeja \& Klessen (2006).

\section{RESULTS}

\subsection{The Large-Scale Structure}

Figures 20 and 2b show the 20th and 35th NN density maps of the PMS stars in the NGC 346 field. While the first is used to identify clusters, the second traces the larger-scale structure, in particular, apart from the bar of N66, it emphasizes a filament (arm-like feature) of enhanced stellar density extending to the north-east from the central association (see Gouliermis et al. 2008) and another one running in east-west direction at the southern edge of the observed area.

Figure 2r shows the 20th NN density of the UMS stars brighter than $m_{V}=18$ in the same field. While the PMS stars show a highly inhomogeneous distribution with several readily identifiable clusters, the UMS stars show a distribution with only one central peak, which roughly coincides with the maximum NN density of the PMS stars.

Figure 3 shows an image of NGC 346 in the [O III] line $(501.1 \mathrm{~nm})$ obtained with the ESO NTT overlaid with the PMS NN density contours. In the central association a high density of both gas and PMS stars is found, but the smaller clusters north and south of the center do not coincide with a higher gas density. The small clusters in the south appear to be aligned offset, but parallel to the gaseous filament. When comparing the PMS stellar distribution to the $8 \mu \mathrm{m}$ observation of the region (Figure 4) a remarkable correlation of the $\mathrm{NN}$ density peaks with the $8 \mu \mathrm{m}$ emission peaks is found for the central association and the clusters north of it.

\subsection{Identified Clusters}

Using the 20th NN density and the $3 \sigma$ limit, ten PMS clusters are detected in NGC 346, labelled A to J from north to south (Fig. 2a).

The central association, Cluster F, is by far the largest cluster with 2349 PMS stars. Clusters A, B, D and E are parts of the filament pointing from the main association towards the north-east more clearly visible in the 35th NN density map (Fig. 2b). Cluster C is a rather evolved open cluster with a PMS population at the age of $\sim 5-15 \mathrm{Myr}$ (Hennekemper et al. 2008), and it may be not related to the much younger other clusters. While Clusters B and D show two clearly visible separate density peaks, they are picked up as single clusters using the $3 \sigma$ limit in the NN density maps. However, when using a $4 \sigma$ threshold, cluster D breaks up into two clusters (D1 and D2), while of cluster B only the western peak remains (B2). Clusters C, D, and E are also identified by Hennekemper et al. (2008) as their Clusters 1, 

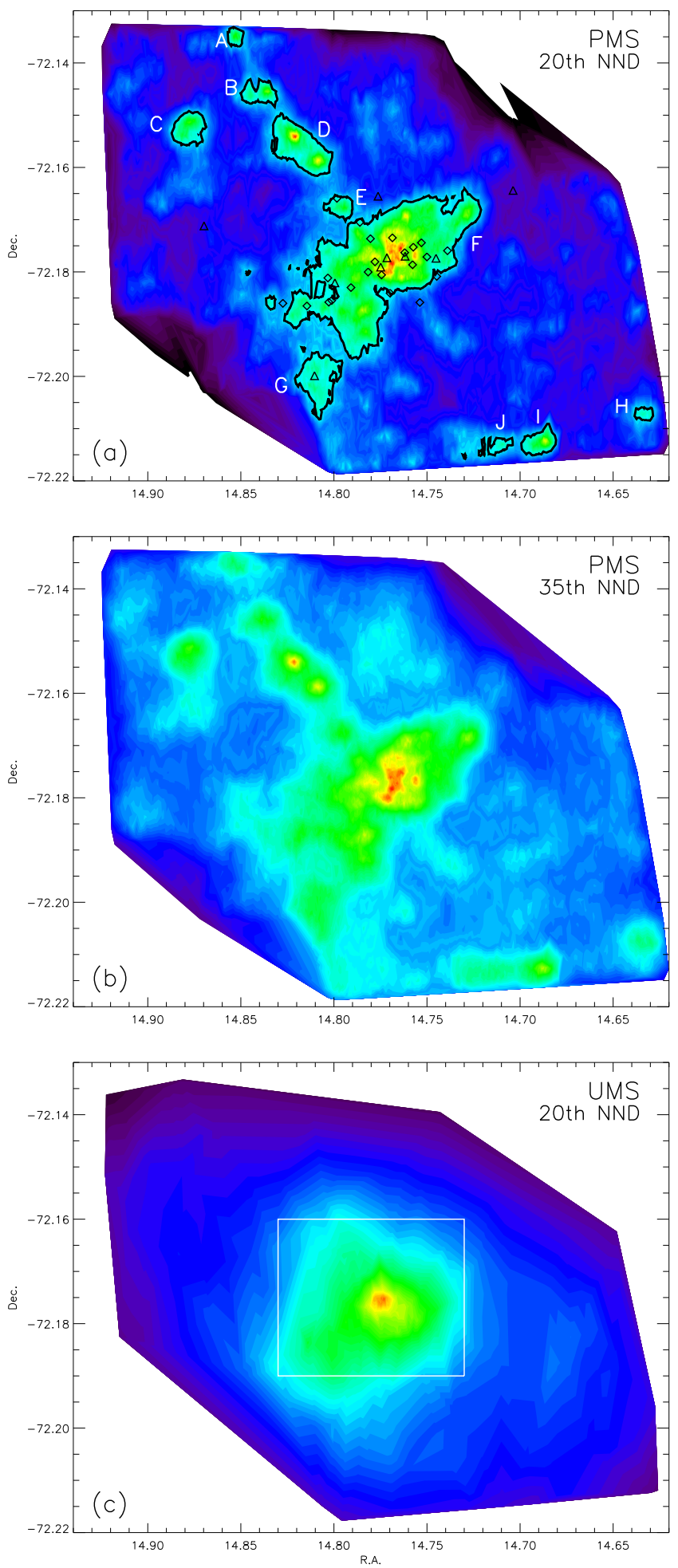

FIG. 2.- NN density maps of NGC 346 (shown in a logarithmic scale): (a) 20th NN density of the PMS stars. The black lines indicate the cluster boundaries, defined as $3 \sigma$ above the average background density. Also shown are the positions of the known $\mathrm{O}$ (diamonds) and B stars (triangles). (b) 35th NN density of the PMS stars, emphasizing the large-scale structure. (c) 20th NN density of the UMS stars brighter than $m_{V}=18$.

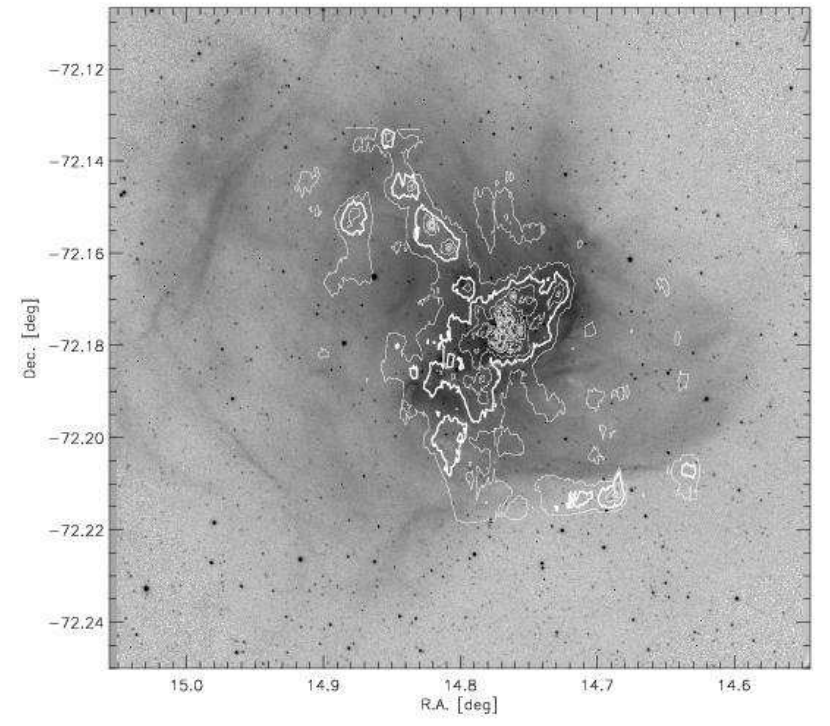

FIG. 3.- NTT [O III] $(501.1 \mathrm{~nm})$ observation of NGC 346 overlaid with the PMS 20th NN density contours; the cluster boundary contour is shown by a thick line.

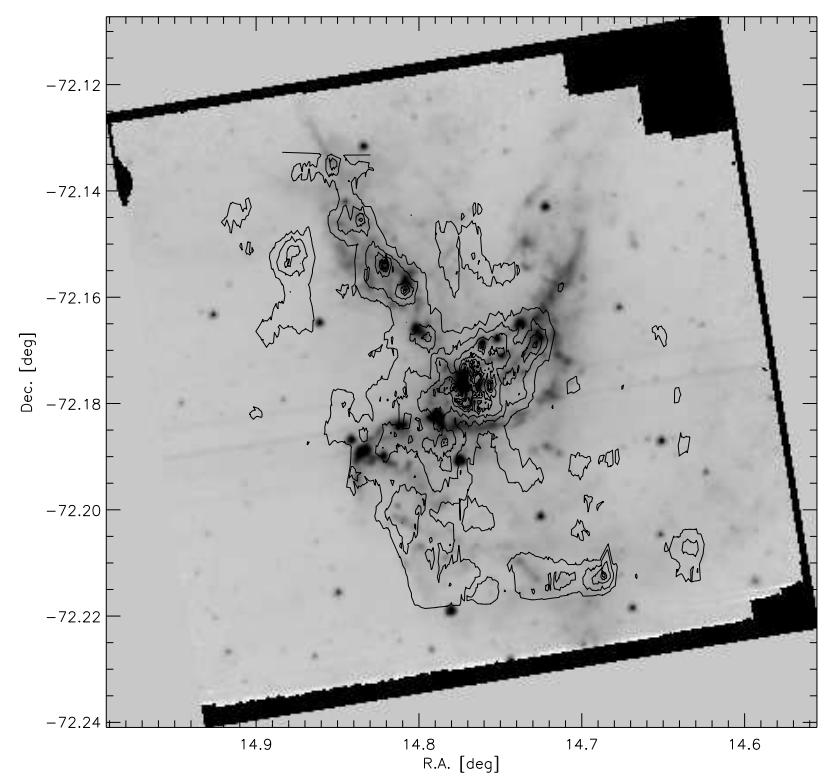

FIG. 4.- Spitzer IRAC Channel $4(8 \mu \mathrm{m})$ observation of NGC 346 overlaid with the PMS 20th NN density contours (analogous to Fig. 3).

2 and 3. Their Clusters 4 and 5 are part of the main association in the NN method. Clusters C, D, and E correspond to the subclusters Sc 16 , Sc $14+$ Sc 15 , and Sc 13 identified by Sabbi et al. (2007), while the central association is broken into eleven subclusters (Sc 1 to Sc 11) by these authors. It is not possible to reproduce the number and locations of the central subclusters of Sabbi et al. (2007) using the NN method even with different $j$ values $(5 \leq j \leq 50)$ and different threshold levels $\left(2 \sigma \leq \rho_{\mathrm{j}} \leq 6 \sigma\right)$.

Table 1 lists the identified PMS clusters with the positions of their 20th NN density centers (columns 2 and 3), the number of cluster members (column 4), their density 
TABLE 1

Detected PMS Clusters

\begin{tabular}{lccrrrr}
\hline Cluster & $\begin{array}{c}\text { RA }(\mathrm{J} 2000) \\
(\mathrm{deg})\end{array}$ & $\begin{array}{c}\text { Dec }(\mathrm{J} 2000) \\
(\mathrm{deg})\end{array}$ & $n_{*}$ & $\begin{array}{c}r_{\mathrm{d}} \\
(\mathrm{pc})\end{array}$ & $\begin{array}{r}\rho_{20}^{\max } \\
\left(\mathrm{pc}^{-2}\right)\end{array}$ & $\mathcal{Q}$ \\
\hline $\mathrm{A}$ & 14.85263 & -72.13482 & 27 & 0.57 & 5.35 & 0.93 \\
$\mathrm{~B}$ & 14.83835 & -72.14563 & 59 & 1.46 & 8.38 & 0.59 \\
$\mathrm{C}$ & 14.87806 & -72.15229 & 90 & 1.79 & 6.11 & 0.74 \\
$\mathrm{D}$ & 14.81802 & -72.15552 & 257 & 2.92 & 36.35 & 0.65 \\
$\mathrm{E}$ & 14.79577 & -72.16745 & 47 & 1.21 & 5.34 & 0.92 \\
$\mathrm{~F}$ & 14.76906 & -72.17724 & 2349 & 5.26 & 40.02 & 0.68 \\
$\mathrm{G}$ & 14.80844 & -72.20081 & 139 & 3.14 & 3.45 & 0.70 \\
$\mathrm{H}$ & 14.63330 & -72.20700 & 18 & 0.70 & 2.49 & 0.86 \\
$\mathrm{I}$ & 14.68797 & -72.21261 & 74 & 1.10 & 13.22 & 0.80 \\
$\mathrm{~J}$ & 14.71087 & -72.21309 & 26 & 1.31 & 2.83 & 0.78 \\
\hline
\end{tabular}
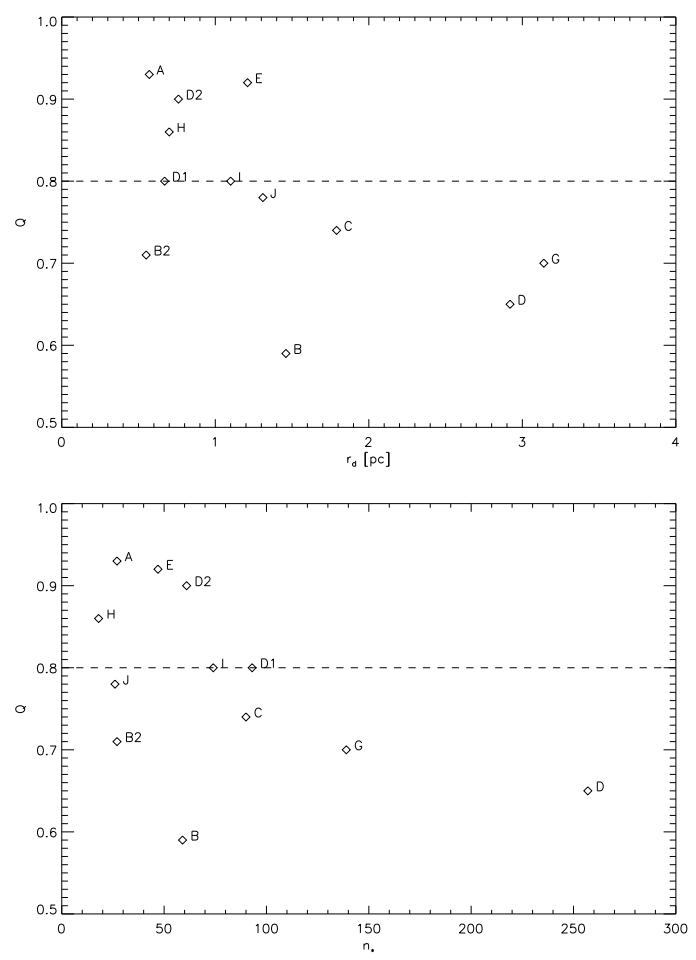

FIG. 5. $-\mathcal{Q}$ versus density radius (upper panel) and number of stars (lower panel) for the identified PMS clusters (cluster F not shown). The dashed line at $\mathcal{Q}=0.8$ indicates the division between hierarchical and centrally condensed clusters.

radius (Eq. 3) in pc (assuming a distance of $62 \mathrm{kpc}$; column 5), maximum 20th NN density (column 6), and $\mathcal{Q}$ (column 7).

\subsection{Clustering Parameters}

The detected clusters show a wide variety in the $\mathcal{Q}$ parameter, ranging from strongly centrally condensed clusters with $\mathcal{Q}>0.9$ (clusters A, E) to highly hierarchical clusters with multiple density peaks and values $\mathcal{Q}<0.7$ (clusters B, D). Also the central association (F) with its several density peaks shows, as expected, a hierarchical distribution $(\mathcal{Q}=0.68)$. However, this value has to be taken with care, since due to a degeneracy of the $\mathcal{Q}$ parameter, highly elongated structures like this one systematically show lower $\mathcal{Q}$ values (Bastian et al. 2008). Applying the correction suggested by Bastian et al. (2008) changes the value to $\mathcal{Q} \approx 0.74$, still in the hierarchical regime. To avoid these uncertainties, and to compare the values for PMS and UMS stars, we use a quadrilateral area around the central region with $14.73 \leq \alpha \leq 14.83$ and $-72.19 \leq \delta \leq-72.16$ (shown as a white box in Fig. 22). Both PMS and UMS stars in this region are equally centrally concentrated $(\mathcal{Q}=0.81)$. As far as the entire NGC 346 area is concerned, the UMS stars show the same $\mathcal{Q}$ value, while the PMS stars show a hierarchical distribution $(\mathcal{Q}=0.73)$.

The subclusters of multi-peak clusters can be centrally concentrated or again hierarchical. The two subclusters D1 and D2 show $\mathcal{Q}$ values of 0.80 and 0.90 , respectively, while $\mathrm{B} 2$ has a value of $\mathcal{Q}=0.71$.

The $\mathcal{Q}$ parameters show a moderate correlation with the size of the cluster measured by $r_{\mathrm{d}}$ and the number of stars, illustrated in Fig. 5. This is in remarkable contradiction to the embedded clusters found in nearby Galactic molecular clouds, where smaller clusters tend to have $\mathcal{Q}$ values well in the hierarchical regime (Schmeja et al. 2008). This may be due to the fact that we are only tracing the more evolved PMS stars in NGC 346 that probably show a more centrally condensed structure than Class $0 / 1$ protostars, which are expected to be hierarchically clustered (Schmeia et al. 2008).

\section{4. $\mathcal{Q}$ versus Magnitude}

We investigate the variation of $\mathcal{Q}$ as a function of magnitude (and thus, mass) for the PMS and UMS sample. This is done for the entire area as well as for the central region. $\mathcal{Q}$ was calculated for sources brighter than a certain magnitude in steps of $0.5 \mathrm{mag}$ as long as the sample contained at least 40 objects. The resulting $\mathcal{Q}$ versus magnitude plots for PMS stars and UMS stars are shown in Fig. 6 and 7, respectively. Both PMS and UMS stars show a decline of $\mathcal{Q}$ with fainter magnitudes when looking at the entire area, indicating that the massive stars are more centrally condensed than lower-mass objects. However, both samples show no correlation of $\mathcal{Q}$ with magnitude when considering the central region.

\subsection{OB Stars}

In Fig. 2a the positions of the spectroscopically verified OB stars in NGC 346 by Massey et al. (1989) are shown overlaid on the 20th NN density map. While it is apparent that the OB stars are centrally concentrated in the association, it is interesting to note that they are not located in the densest (yellow-red) regions of PMS stars. This indicates that the density peaks of PMS stars observed in Cluster F may be an observational bias, due to the fact that the OB stars outshine the faint PMS stars in their vicinity. This provides further support to our identification of the association as a single, not sub-clustered system from both observed UMS and PMS populations. As far as the clustering behavior of the OB stars alone is concerned, they do not seem to form any central condensation. Indeed, when considering the $27 \mathrm{OB}$ stars lying in the association, they show a distribution in the hierarchical range with $\mathcal{Q}=0.77$.

\section{DISCUSSION AND CONCLUSIONS}

\subsection{Theoretical Concepts}

With the advancement of infrared detectors and the ability to do wide-area surveys, it has been recognized 

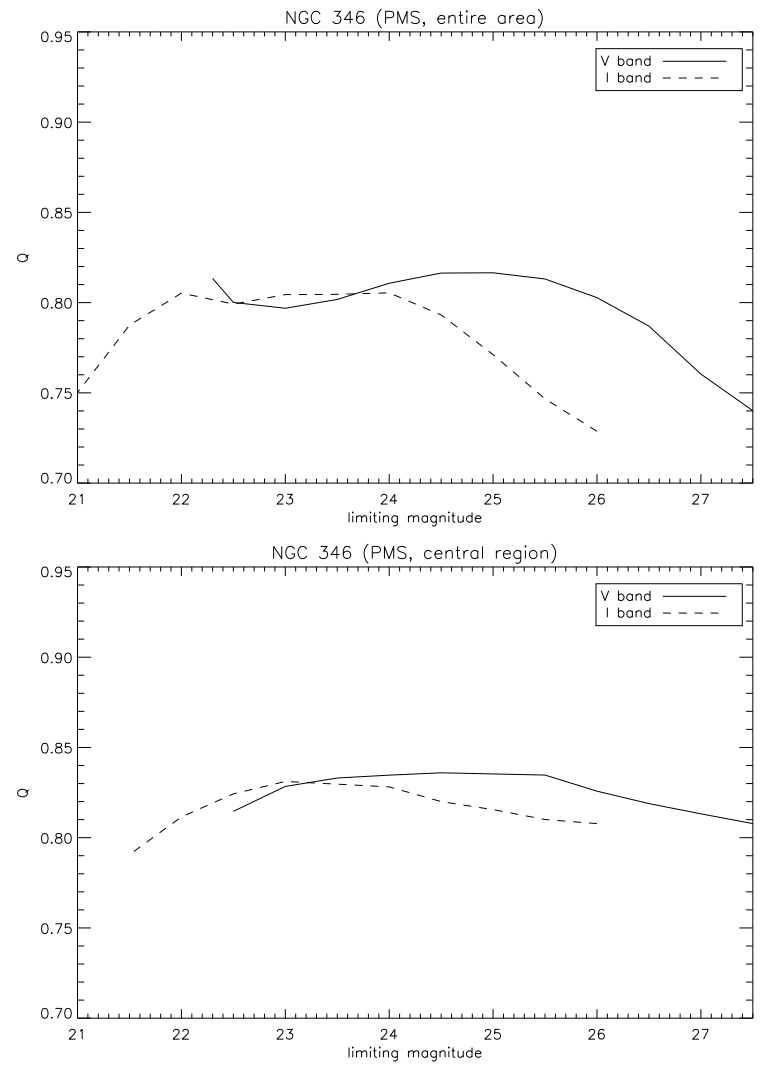

FIG. 6. $-\mathcal{Q}$ versus limiting magnitude for the PMS stars in the entire area (upper panel) and in the central region (lower panel).

that a large fraction of all stars in the Milky Way form in clusters and aggregates of various size and mass scales and that isolated or widely distributed star formation is the exception rather than the rule (Lada \& Lada 2003). It is therefore highly interesting to study the clustering behavior in neighboring galaxies as well. The complex hierarchical structure of molecular clouds provides a natural explanation for clustered star formation. Molecular clouds vary enormously in size and mass. In small, lowdensity, clouds stars form with low efficiency, more or less in isolation or scattered around in small groups of up to a few dozen members. Denser and more massive clouds may build up stars in associations and clusters of a few hundred members. This appears to be the most common mode of star formation, at least in the solar neighborhood (Adams \& Myers 2001). The formation of dense rich clusters with thousands of stars or more is rare. The cluster NGC 346, therefore, is a very extreme case of clustered star formation.

Star formation is governed by the complex interplay between gravitational attraction in molecular clouds and any opposing processes such as supersonic turbulence, thermal pressure and magnetic fields.

There are two main competing models that describe the evolution of the cloud cores. It was proposed in the 1980's that cores in low-mass star-forming regions evolve quasi-statically in magnetically subcritical clouds (Shu et al. 1987). Gravitational contraction is mediated by ambipolar diffusion (Mouschovias \& Spitzer 1976; Mouschovias 1991) causing a redistribution of magnetic
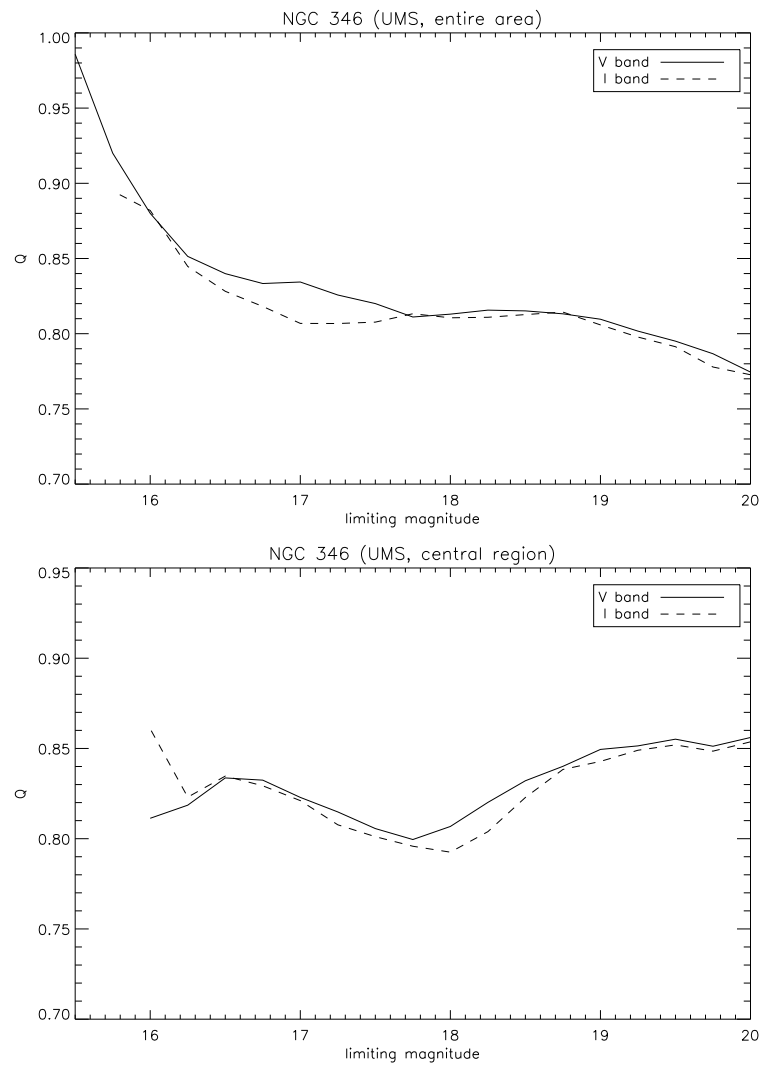

FIG. 7.- $\mathcal{Q}$ versus limiting magnitude for the UMS stars in the entire area (upper panel) and in the central region (lower panel).

flux until the inner regions of the core become supercritical and go into dynamical collapse. Measurements of the Zeeman splitting of molecular lines in nearby cloud cores, however, indicate magnetic field strengths that fall below the critical value, in some cases only by a small margin, in many cases however, by factors of many (Crutcher 1999; Bourke et al. 2001; Crutcher et al. 2008). Magnetic fields therefore seem not to be the dominant agent regulating stellar birth.

This has lead to the suggestion that the supersonic turbulence ubiquitously observed in molecular clouds is the main physical process governing star formation (Mac Low \& Klessen 2004; Ballesteros-Paredes et al. 2007; McKee \& Ostriker 2007). Supersonic turbulence plays a dual role. On large scales it can support clouds against contraction, however, on small scales it can provoke localized collapse. Turbulence establishes a complex network of interacting shocks, where dense cores form at the stagnation points of convergent flows. The density can be large enough for gravitational collapse to set in. However, the fluctuations in turbulent velocity fields are highly transient. The random flow that creates local density enhancements can disperse them again. For local collapse to actually result in the formation of stars, high density fluctuations must collapse on time scales shorter than the typical time interval between two successive shock passages. Only then are they able to 'decouple' from the ambient flow and survive subsequent shock interactions. The shorter the time between shock passages, the less likely these fluctuations are to survive. 
Hence, the timescale and efficiency of protostellar core formation depend strongly on the wavelength and strength of the driving source (Klessen et al. 2000; Heitsch et al. 2001; Vázquez-Semadeni et al. 2003), and accretion histories of individual protostars are strongly time varying (Klessen 2001; Schmeja \& Klessen 2004).

Interstellar turbulence is observed to be dominated by large-scale velocity modes (Mac Low \& Ossenkopf 2000; Ossenkopf et al. 2001; Ossenkopf \& Mac Low 2002). This implies it is very efficient in sweeping up molecular cloud material, creating massive coherent structures which can go into large-scale gravitational collapse. In the extreme case, dense clusters with many thousand stars build up on one or two crossing times (Hartmann et al. 2001; Elmegreen 2000, 2007). Numerical simulations of gravoturbulent cloud fragmentation indicate that in such cases star clusters would build up in a hierarchical fashion with sub-clusters which evolve dynamically and merge together to build up the final, centrally-condensed cluster Klessen \& Burkert 2000; Clarke et al. 2000; Bonnell et al. 2003; Clark \& Bonnell 2005; Bonnell \& Bate 2006), with stellar feedback strongly influencing this process (Krumholz et al. 2007). Schmeja \& Klessen (2006) investigated the temporal evolution of a large set of numerical simulations of gravoturbulent fragmentation and showed that the $\mathcal{Q}$ parameter of all resulting clusters increases with time. This is confirmed by observations of embedded clusters in nearby molecular clouds, where the younger Class $0 / 1$ sources show substantially lower $\mathcal{Q}$ values than the more evolved Class 2/3 objects (Schmeja et al. 2008).

\subsection{The Structure of NGC 346/N66}

Within our investigation of the spatial distribution of PMS stars in the NGC 346/N66 region using the nearest neighbor and minimum spanning tree methods an increased NN density of PMS stars is found in the central association and in two filamentary structures, one extending to the north-east from the center of N66, and another at the southern edge of the observed area, extending from east to west. We identify ten individual clusters of PMS stars in the region, with the central association being the largest. While several PMS density peaks are found within the association NGC 346, the system could not be resolved into individual subclusters. Our methods show evidence of centrally concentrated UMS stars and PMS stars in a hierarchical distribution. The PMS density peaks of the association coincide with peaks of both [O III] and $8 \mu \mathrm{m}$ emission, indicating that the system is in an early evolutionary stage.

The identified PMS clusters in the whole area of NGC 346/N66 show a wide range of morphologies from hierarchical multi-peak configurations to centrally condensed clusters. Only about $40 \%$ of the PMS stars in the investigated area belong to the ten identified clusters. The remaining $60 \%$ of PMS stars is found concentrated in small (statistically insignificant) groups and mostly dispersed over the entire area. This fraction of clustered PMS stars is comparable to that found in Galactic starforming regions. In their SCUBA survey of the Perseus molecular cloud Hatchell et al. (2005) found that 40 $60 \%$ of the protostars and prestellar cores are located in small clusters $\left(<50 \mathrm{M}_{\odot}\right)$ and isolated objects. Investigations in other Galactic star-forming regions like
Ophiuchus, Orion and Monoceros yield a percentage of distributed populations between 20 and $60 \%$ with a "typical" value of 25\% (Carpenter 2000; Allen et al. 2007). However, there are uncertainties due to the incompleteness of the samples and the definition of the clusters. Moreover, the clustering properties themselves depend on the age of the population considered and on the masses of the stars. As a consequence, it is very difficult to compare and interpret these numbers.

Based on the analysis of $\mathcal{Q}$ versus magnitude, both PMS and UMS stars show roughly the same degree of central concentration over the entire magnitude range. When we consider the entire area, $\mathcal{Q}$ is found to decrease with magnitude, providing evidence that both PMS and UMS stars are centrally concentrated in respect to the whole observed region of NGC 346. This, especially for the bright stars, is expected considering that the central association contains the largest fraction of stars. However, when we consider only the central region of the area, $\mathcal{Q}$ does not seem to depend strongly on magnitude, and therefore no evidence of mass segregation in the central association is found.

Whether the clusters in NGC 346 have formed coevally (Sabbi et al. 2007) or in a sequential process over several Myr (Massey et al. 1989; Rubio et al. 2000) is still under debate. As young clusters seem to evolve from a hierarchical configuration to a centrally concentrated one with time, the fact that the identified clusters in NGC 346 show a wide range in their structures with $\mathcal{Q}$ values ranging from 0.65 to 0.93 , may indeed indicate that they are at different evolutionary stages. However, the absolute $\mathcal{Q}$ values are not correlated with the cluster age, but probably depend on a variety of additional factors like initial conditions or turbulent energy in the cloud (Schmeja et al. 2008), and therefore this is no direct proof that the clusters have formed at different times. However, the rather high $\mathcal{Q}$ values of the southern clusters (H, I, J) around or above 0.8 agree with other age indicators. While the peaks of the central concentration and the clusters to the north of it coincide with the peaks of dust emission (indicated by the $8 \mu \mathrm{m}$ observations) and gas (traced by [O III]), the southern clusters appear to be associated with less amounts of gas and dust, suggesting an older age of these clusters. Hennekemper et al. (2008) find that the northern clusters comprise a younger PMS population (not older than 2.5 Myr) than the other clusters. Recently, Gouliermis et al. (2008) presented evidence that these clusters are the product of triggered star formation. In combination, all these indications strongly suggest that the identified PMS clusters are not the product of a single star formation event.

In conclusion our investigation of the spatial distribution of PMS stars in the region of NGC 346/N66 using the nearest neighbor and minimum spanning tree methods showed that this region appears to be the product of hierarchical star formation, where the individual clusters show different structures according to their size, evolutionary stage, position in the cloud and possible additional internal or environmental factors. The overall structure and statistical properties of the region are consistent with numerical calculations of gravoturbulent cloud fragmentation (e.g. Klessen et al. 2000; Clark et al. 2005; Bonnell \& Bate 2006). NGC 346/N66 is a unique case, where the complexity of star formation 
can be observed in its entire length-scale.

SS and DAG are supported by the Deutsche Forschungsgemeinschaft (DFG) through individual grants SCHM 2490/1-1 and GO 1659/1-1, respectively. RSK acknowledges partial support from the DFG via grant KL 1358/1-3 and via the priority program SFB 439 Galaxies in the Young Universe. This work is based on observations made with the NASA/ESA Hubble Space Telescope, obtained from the data archive at the
Space Telescope Science Institute (STScI). STScI is operated by the Association of Universities for Research in Astronomy, Inc. under NASA contract NAS 5-26555. This work also makes use of observations made with the ESO NTT at La Silla Observatory under program ID 56.C-0379 and observations made with the Spitzer Space Telescope, which is operated by the Jet Propulsion Laboratory, California Institute of Technology, under a contract with NASA.

\section{REFERENCES}

Adams, F. C., \& Myers, P. C. 2001, ApJ, 553, 744

Allen, L., et al. 2007, in Protostars and Planets V, ed. B. Reipurth, D. Jewitt, \& K. Keil (Tucson: Univ. Arizona Press), 361

Ballesteros-Paredes, J., Klessen, R. S., Mac Low, M.-M., \& Vázquez-Semadeni, E. 2007, in Protostars and Planets V, ed. B. Reipurth, D. Jewitt, \& K. Keil (Tucson: Univ. Arizona Press), 63

Bastian, N., Gieles, M., Ercolano, B., \& Gutermuth, R. 2008, MNRAS, in press (arXiv:0810.3190)

Bica, E. L. D., \& Schmitt, H. R. 1995, ApJS, 101, 41

Bolatto, A., et al. 2007, ApJ, 655, 212

Bonnell, I. A., \& Bate, M. R. 2006, MNRAS, 370, 488

Bonnell, I. A., Bate, M. R., \& Vine, S. G. 2003, MNRAS, 343, 413

Borůvka, O. 1926, Práce moravské přírodovědecké společnosti, 3, 37

Bourke, T. L., Myers, P. C., Robinson, G., \& Hyland, A. R. 2001, ApJ, 554, 916

Briceño, C., Preibisch, T., Sherry, W. H., Mamajek, E. A., Mathieu, R. D., Walter, F. M., \& Zinnecker, H. 2007, in Protostars and Planets V, ed. B. Reipurth, D. Jewitt, \& K. Keil (Tucson: Univ. Arizona Press), 345

Carpenter, J. M. 2000, AJ, 120, 3139

Cartwright, A., \& Whitworth, A. P. 2004, MNRAS, 348, 589

Casertano, S., \& Hut, P. 1985, ApJ, 298, 80

Clark, P. C., \& Bonnell, I. A. 2005, MNRAS, 361, 2

Clark, P. C., Bonnell, I. A., Zinnecker, H., \& Bate, M. R. 2005, MNRAS, 359, 809

Clarke, C. J., Bonnell, I. A., \& Hillenbrand, L. A. 2000, in Protostars and Planets IV, ed. V. Mannings, A. P. Boss, \& S. S. Russell (Tucson: Univ. Arizona Press), 151

Contursi, A., et al. 2000, A\&A, 362, 310

Crutcher, R. M. 1999, ApJ, 520, 706

Crutcher, R. M., Hakobian, N., \& Troland, T. H. 2008, ApJ, accepted (arXiv:0807.2862)

Danforth, C. W., Sankrit, R., Blair, W. P., Howk, J. C., \& Chu, Y.-H. 2003, ApJ, 586, 1179

Davies, R. D., Elliott, K. H., \& Meaburn, J. 1976, MmRAS, 81, 89

de Boer, K. S., \& Savage, B. D. 1980, ApJ, 238, 86

Dolphin, A. E. 2000, PASP, 112, 1383

Efremov, Y. N., \& Elmegreen, B. G. 1998, MNRAS, 299, 588

Elmegreen, B. G. 2000, ApJ, 530, 277

Elmegreen, B. G. 2006, in Globular Clusters, Guide to Galaxies, ed. T. Richtler et al. (Berlin: ESO/Springer), in press (astro-ph/0605519)

Elmegreen, B. G. 2007, ApJ, 668, 1064

Elmegreen, B. G., Efremov, Y., Pudritz, R. E., \& Zinnecker H. 2000, in Protostars and Planets IV, ed. V. Mannings, A. P. Boss, \& S. S. Russell (Tucson: Univ. Arizona Press), 179

Evans, C. J., Lennon, D. J., Smartt, S. J., \& Trundle, C. 2006, A\&A, 456, 623

Gouliermis, D. A., Dolphin, A. E., Brandner, W., \& Henning, T. 2006, ApJS, 166, 549

Gouliermis, D. A., Chu, Y.-H., Henning, T., Brandner, W. Gruendl, R. A., Hennekemper, E., \& Hormuth, F. 2008, ApJ, 688,1050

Hartmann, L., Ballesteros-Paredes, J., \& Bergin, E. A. 2001, ApJ, 562,852

Hatchell, J., Richer, J. S., Fuller, G. A., Qualtrough, C. J., Ladd, E. F., \& Chandler, C. J. 2005, A\&A, 440, 151
Henize, K. G. 1956, ApJS, 2, 315

Hennekemper, E., Gouliermis, D. A., Henning, T., Brandner, W., \& Dolphin, A. E. 2008, ApJ, 672, 914

Heitsch, F., Mac Low, M.-M., \& Klessen, R. S. 2001, ApJ, 547, 280

Hunter, I., et al. 2008, A\&A, 479, 541

Kennicutt, R. C., Jr. 1988, ApJ, 334, 144

Klessen, R. S. 2001, ApJ, 550, L77

Klessen, R. S., \& Burkert, A. 2000, ApJS, 128, 287

Klessen, R. S., Heitsch, F., \& Mac Low, M.-M. 2000, ApJ, 535, 887

Krumholz, M. R., Klein, R. I., \& McKee, C. F. 2007, ApJ, 656, 959

Kruskal, J. B. Jr. 1956, Proc. Amer. Math. Soc., 7, 48

Lada, C. J., \& Lada, E. A. 2003, ARA\&A, 41, 57

Laney, C. D., \& Stobie, R. S. 1994, MNRAS, 266, 441

Le Coarer, E., Rosado, M., Georgelin, Y. P., Viale, A., \& Goldes, G., 1993, A\&A, 280, 365

Mac Low, M.-M., \& Ossenkopf, V. 2000, A\&A, 353, 339

Mac Low, M.-M., \& Klessen, R. S. 2004, Rev. Mod. Phys., 76, 125

Massey, P., Parker, J. W., \& Garmany, C. D. 1989, AJ, 98, 1305

McKee, C. F., \& Ostriker, E. C. 2007, ARA\&A, 45, 565

Mouschovias, T. C. 1991, NATO ASIC Proc. 342: The Physics of

Star Formation and Early Stellar Evolution, 61

Mouschovias, T. C., \& Spitzer, L., Jr. 1976, ApJ, 210, 326

Nazé, Y., et al. 2002, ApJ, 580, 225

Nazé, Y., Manfroid, J., Stevens, I. R., Corcoran, M. F., \& Flores, A. 2004, ApJ, 608, 208

Niemela, V. S., Marraco, H. G., \& Cabanne, M. L. 1986, PASP, 98,1133

Ossenkopf, V., \& Mac Low, M.-M. 2002, A\&A, 390, 307

Ossenkopf, V., Klessen, R. S., \& Heitsch, F. 2001, A\&A, 379, 1005

Prim, R. C. 1957, Bell Syst. Tech. J., 36, 1389

Reid, W. A., et al. 2006, MNRAS, 367, 1379

Rochau, B., Gouliermis, D. A., Brandner, W., Dolphin, A. E., \& Henning, T. 2007, ApJ, 664, 322

Rubio, M., et al. 2000, A\&A, 359, 1139

Sabbi, E., et al. 2007, AJ, 133, 44

Scalo, J. M. 1985, in Protostars and Planets II, ed. D. C. Black \& M. S. Matthews (Tucson: Univ. Arizona Press), 201

Schmeja, S., \& Klessen, R. S. 2004, A\&A, 419, 405

Schmeja, S., \& Klessen, R. S. 2006, A\&A, 449, 151

Schmeja, S., Kumar, M. S. N., \& Ferreira, B. 2008, MNRAS, 389, 1209

Shu, F. H., Adams, F. C., \& Lizano, S. 1987, ARA\&A, 25, 23

Simon, J. D., et al. 2007, ApJ, 669, 327

Stahler, S. W., \& Palla, F. 2005, The Formation of Stars (Weinheim: Wiley-VCH)

Staveley-Smith, L., Sault, R. J., Hatzidimitriou, D., Kesteven, M. J., \& McConnelle, D. 1997, MNRAS, 289, 225

Vázquez-Semadeni, E. 2004, Ap\&SS, 292, 187

Vázquez-Semadeni, E., Ballesteros-Paredes, J., \& Klessen, R. S. 2003, ApJ, 585, L131

von Hoerner, S. 1963, ZAp, 57, 47

Walborn, N. R., et al. 2000, PASP, 112, 1243

Ye, T., Turtle, A. J., \& Kennicutt, R. C. Jr. 1991, MNRAS, 249, 722 